\title{
EMBEDDING SEMIRINGS IN SEMIRINGS WITH MULTIPLICATIVE UNIT
}

\author{
K. R. PEARSON 1
}

(Received 28 November 1966)

A topological semiring is a system $(S,+, \cdot)$ where $(S,+)$ and $(S, \cdot)$ are topological semigroups and - distributes across + as in a ring; that is, for all $x, y, z$ in $S$,

$$
\begin{aligned}
x \cdot(y+z) & =(x \cdot y)+(x \cdot z), \\
(x+y) \cdot z & =(x \cdot z)+(y \cdot z) .
\end{aligned}
$$

The operations + and $\cdot$ are called addition and multiplication respectively.

If $S$ is any topological semiring and we adjoin to $S$ an element 0 as an isolated point and let 0 be a multiplicative zero and either an additive unit or an additive zero for $S^{\prime}=S \cup\{0\}$, then it can be easily seen that $S^{\prime}$ is also a topological semiring. Thus it is always possible to embed $S$ in a semiring $S^{\prime}$ with multiplicative zero; also $S^{\prime}$ is compact when $S$ is.

Selden has shown in Theorem 7 of [6] (see also [7]) that each additive group in a compact semiring with multiplicative unit must be totally disconnected. This means that the semiring $(C,+, \cdot)$, where $(C,+)$ is the circle group and $x \cdot y=0$ for all $x, y \in C$, cannot be embedded in a compact semiring with multiplicative unit. We investigate here conditions under which it is possible to embed a semiring in a semiring with multiplicative unit. In particular, we derive in Theorem 3 a necessary and sufficient condition for the embedding of a compact additively commutative semiring in a compact semiring with multiplicative unit to be possible. The special case of embedding a compact ring in a compact ring with unit is also dealt with.

It is first necessary to establish some points of notation. If $x$ is a member of an additive semigroup and $n$ is a positive integer, we shall use $n x$ to mean the semigroup sum of $n$ elements each equal to $x$. Note that if 1 is a multiplicative left unit of a semiring then $n$ denotes the semiring sum of $n$ elements each equal to 1 . Hence $n x$ also equals the product of $n$ and $x$. For any $x$ in $(S,+, \cdot)$, let Michael.

1 This paper is part of the author's Ph.D. thesis, written under the supervision of Dr. J. H. 


$$
\begin{aligned}
0[+](x) & =\{n x \mid n \text { a positive integer }\}, \\
\Gamma[+](x) & =0[+](x)^{-}, \\
K[+](x) & =\bigcap_{n=1}^{\infty}\{m x \mid m \geqq n\}^{-},
\end{aligned}
$$

where - denotes topological closure. Then $\Gamma[+](x)$ is a commutative (additive) semigroup. If it is compact, then $K[+](x)$ is the minimal ideal of $\Gamma[+](x)$ and is also the maximal additive group in $\Gamma[+](x)$ (see $[5]$, Theorem 3.1.1 or [2], Theorems 3.3 and 3.4). A semiring is said to be additively $\Gamma$-compact if $\Gamma[+](x)$ is compact for each $x$ in $S$.

$S$ is said to be a subsemiring of a topological semiring $T$ if and only if for each $x, y$ in $S$, both $x+y$ and $x \cdot y$ are in $S ; S$, given the relative topology, is a topological semiring. We shall say that a topological semiring $S$ can be embedded in a topological semiring $T$ if there is a subsemiring $S_{1}$ of $T$ which is topologically isomorphic with $S$; note that $S_{1}$ need not be topologically closed in $T$.

If $S$ is an additively $\Gamma$-compact subsemiring of a topological semiring $T$ then, for each $x$ in $S$, the closure of $0[+](x)$ in the relative topology of $S$ is compact so that it is also compact in the topology of $T$. Because $T$ is Hausdorff, it follows that the closure of $0[+](x)$ in the relative topology of $S$ is the same as its closure in the topology of $T$. Thus we can use the symbol $\Gamma[+](x)$ without fear of confusion.

The first result is a topological extension of a familiar construction (see, for example, [4], page 49).

TheOREM 1. Any additively commutative topological semiring $S$ can be embedded in some additively commutative topological semiring $T$ with multiplicative unit. Further, $T$ can be made locally compact when $S$ is locally compact.

Proof. We first adjoin to $S$ an element $\alpha$ as an isolated point and let $\alpha$ be an additive unit and a multiplicative zero for $S^{\prime}=S \cup\{\alpha\}$. Let $N$ denote the (locally compact) semiring of non-negative integers with ordinary addition and multiplication. Then we put $T=N \times S^{\prime}$ and define addition * and multiplication $\circ$ on $T$ by

$$
\begin{aligned}
& \left(n_{1}, x_{1}\right) *\left(n_{2}, x_{2}\right)=\left(n_{1}+n_{2}, x_{1}+x_{2}\right), \\
& \left(n_{1}, x_{1}\right) \circ\left(n_{2}, x_{2}\right)=\left(n_{1} n_{2}, x_{1} x_{2}+n_{1} x_{2}+n_{2} x_{1}\right),
\end{aligned}
$$

where $0 x=\alpha$ for all $x$ in $S^{\prime}$. It is a simple matter to check that $(T, *, 0)$ is a topological semiring which is locally compact when $S$ is locally compact. Also $(1, \alpha)$ is a multiplicative unit for $T$ and $\{0\} \times S$ is a subsemiring topologically isomorphic with $S$.

In what follows we are concerned with additively $\Gamma$-compact semirings. 
For each $x, \Gamma[+](x)$ is then a compact monothetic semigroup and the structure of all such semigroups, first found by Hewitt in [2], is given in Theorems 3.1.6 and 3.1.7 of [5]. In broad outline, our procedure is to construct what is roughly speaking the largest relevant compact monothetic semigroup and use it in a cartesian product with $S^{\prime}$ (as in Theorem 1) to be the space in which $S$ is embedded when $S$ is additively commutative.

LeMma 1. If $S$ is a topological semiring then $\Gamma[+](x)$ is a subsemiring if and only if $x^{2} \in \Gamma[+](x)$.

Proof. Suppose $x^{2} \in \Gamma[+](x)$. Then if $p, q$ are positive integers, $(p q) x^{2} \in \Gamma[+](x)$; but $(p q) x^{2}=(p x)(q x)$ and so $(p x)(q x) \in \Gamma[+](x)$. Hence $\{0[+](x)\}^{2} \subset 0[+](x)$ and it follows from the continuity of multiplication that $\{\Gamma[+](x)\}^{2} \subset \Gamma[+](x)$.

LeMma 2. If $S$ is a topological semiring and $x$ is a multiplicative idempotent, then $\Gamma[+](x)$ is a subsemiring for which $x$ is a multiplicative unit.

Proof. It follows from Lemma 1 that $\Gamma[+](x)$ is a subsemiring. Also, for any positive integer $m,(m x) \cdot x=m x^{2}=m x=x \cdot(m x)$. Thus $x$ is a multiplicative unit for $0[+](x)$ and hence for $\Gamma[+](x)$.

LEMma 3. Let $S$ be a topological semiring containing an element $x$ for which $\Gamma[+](x)$ is compact. Then for each $y$ in $S$,

(i) $y \cdot \Gamma[+](x)=\Gamma[+](y x)$ and $\Gamma[+](x) \cdot y=\Gamma[+](x y)$;

(ii) $y \cdot K[+](x)=K[+](y x)$ and $K[+](x) \cdot y=K[+](x y)$.

Proof. The mapping $\phi: S \rightarrow S$ given by $\phi(z)=y z$ is continuous and $\phi(0[+](x))=0[+](y x)$. It follows from the compactness of $0[+](x)^{-}$that

$$
\phi\left(0[+](x)^{-}\right)=0[+](y x)^{-}=\Gamma[+](y x)
$$

(see Corollary 2, page 101 and Prop. 9, page 61 of $[1])$. Hence $\Gamma[+](y x)$ is compact and so $K[+](y x)$ exists. Because $K[+](x)$ is an additive group and $\phi$ is an additive homomorphism onto $y \cdot K[+](x)$, we see that $y \cdot K[+](x)$ is an additive group, so that $y \cdot K[+](x) \subset K[+](y x)$, the maximal additive group in $\Gamma[+](y x)$. On the other hand, if $z \in \Gamma[+](y x)$ and $w \in y \cdot K[+](x)$, then there are $z_{1}, w_{1}$ in $\Gamma[+](x), K[+](x)$ respectively with $z=y z_{1}$, $w=y w_{1}$, and therefore $z+w=y\left(z_{1}+w_{1}\right) \in y \cdot K[+](x)$ since $K[+](x)$ is an additive ideal of $\Gamma[+](x)$. Thus $y \cdot K[+](x)$, being an additive ideal of $\Gamma[+](y x)$, contains the minimal such ideal $K[+](y x)$, and the result follows.

We now make the construction previously referred to.

ExAmple. Let $P$ be the set of prime integers and put

$$
N_{1}=\underset{\rho \in P}{X} \Delta_{\rho}
$$


where, for each $\rho \in P, \Delta_{\rho}$ is the (compact) ring of $\rho$-adic integers (see, for example, [3], $\S 10$ ) and $N_{1}$ is given the product topology. If we give $N_{1}$ coordinate-wise addition and multiplication, $N_{1}$ is a compact ring. For any $\rho \in P$ we let $0_{\rho}$ be the additive unit and $1_{\rho}$ be the multiplicative unit of $\Delta_{\rho}$. Then let $u \in N_{1}$ be such that $u_{\rho}$, the coordinate of $u$ in $\Delta_{\rho}$, is equal to $1_{\rho}$ for all $\rho \in P$. Clearly $u$ is a multiplicative unit for $N_{1}$. It also follows that $\left\{n u \mid n\right.$ a positive integer\} is dense in $N_{1}$; we prove this in Lemma 4 below. Thus the additive group of $N_{1}$ is monothetic with generator $u$.

Let $N_{2}$ be the set of positive integers and put $N_{3}=N_{1} \cup N_{2}$. We can make $N_{3}$ a compact monothetic additive semigroup by proceeding as in Theorem 3.1.7 of [5] (see also Theorem 5.3 of [2]). We take addition in $N_{2}$ to be ordinary addition, put

$$
x+m=x+m u=m+x \text { if } x \in N_{1} \text { and } m \in N_{2},
$$

and retain the same addition in $N_{1}$. We define a topology on $N_{3}$ by letting each point in $N_{2}$ be isolated and, for each $x$ in $N_{1}$, we take

$$
\left\{\begin{array}{l|l}
V_{n}^{*}(x) & \begin{array}{l}
V_{n}^{*}(x)=V(x) \cup\{m \mid m \geqq n \text { and } m u \in V(x)\}, \text { where } \\
V(x) \text { is any neighbourhood of } x \text { in } N_{1} \text { and } n \geqq 1
\end{array}
\end{array}\right\}
$$

as the set of all neighbourhoods of $x$. It is shown in [5], Theorem 3.1.7 (see also [2], Theorem 5.3) that $N_{3}$ is a compact additive semigroup with $N_{2}^{-}=\Gamma[+](1)=N_{3}$ and $K[+](1)=N_{1}$.

Finally we make $N_{3}$ a semiring by taking ordinary multiplication on $N_{2}$, putting

$$
m \cdot x=x \cdot m=m x \quad \text { if } \quad x \in N_{1}, m \in N_{2},
$$

and retaining the multiplication on $N_{1}$. It is not difficult to check that $N_{3}$ becomes a compact semiring with multiplicative unit $\mathbf{l}$.

Lemma 4. Let $u$ and $N_{1}$ be as in the example above. Then $\Gamma[+](u)=N_{1}$.

Proof. It follows from Theorem 25.16 of [3] that there is an element $v$ of $N_{1}$ such that

$$
\{n v \mid n \text { any integer }\}
$$

is dense in $N_{1}$ and so, as shown on page 109 of [5] (see also $\S 2$ of [2]), $\Gamma[+](v)=N_{1}$. For any $\rho \in P$, let $v_{\rho}$ be the coordinate of $v$ which is in $\Delta_{\rho}$. Because $\Gamma[+]\left(v_{\rho}\right)$ must be $\Delta_{\rho}$, it follows from Lemma 3 that

$$
\Delta_{\rho}=\Gamma[+]\left(v_{\rho}\right)=\Gamma[+]\left(1_{\rho} \cdot v_{\rho}\right)=\Gamma[+]\left(1_{\rho}\right) \cdot v_{\rho}=\Delta_{\rho} \cdot v_{\rho} .
$$

since $\Gamma[+]\left(1_{\rho}\right)=\Delta_{\rho}$ (see $\S 10.6$ of $\left.[3]\right)$. Thus there exists an element $w_{\rho}$ of $\Delta_{\rho}$ with $1_{\rho}=w_{\rho} v_{\rho}$. Let $w$ be the element of $N_{1}$ which has its coordinate in $\Delta_{\rho}$ equal to $w_{\rho}$ for all $\rho \in P$; then $w v=u$. Hence, since $u$ is a multiplicative unit for $N_{1}$, 


$$
N_{1}=u N_{1}=(w v) N_{1}=w\left(v N_{1}\right) \subset w N_{1} \subset N_{1}
$$

and we see that $w N_{1}=N_{1}$. Thus, by Lemma 3,

$$
\Gamma[+](u)=\Gamma[+](w v)=w \Gamma[+](v)=w N_{1}=N_{1} .
$$

Our main effort is devoted to proving the following theorem.

THEOREM 2. Let $S$ be an additively commutative and additively $\Gamma$-compact semiring. The following are equivalent.

(i) $S$ can be embedded in an additively commutative and additively $\Gamma$ compact semiring with multiplicative unit.

(ii) $S$ can be embedded in an additively $\Gamma$-compact semiring with multiplicative left unit.

(iii) There is a continuous extension $\Psi: N_{3} \times S \rightarrow S$ of the mapping $\psi: N_{2} \times S \rightarrow S$ defined by $\psi(n, x)=n x$ for $n \in N_{2}, x \in S$.

Clearly (i) implies (ii). We shall prove the theorem by showing that (ii) $\Rightarrow$ (iii) and (iii) $\Rightarrow$ (i). That (ii) $\Rightarrow$ (iii) follows from the following more general result.

Lemma 5. Let $S$ be any additively $\Gamma$-compact subsemiring of a topological semiring $T$ with multiplicative left unit $\alpha$ such that $\Gamma[+](\alpha)$ is compact. Then there is a continuous additive extension $\Psi: N_{3} \times S \rightarrow S$ of the mapping $\psi: N_{2} \times S \rightarrow S$ defined by $\psi(n, x)=n x$. (Note that $\Psi$ is uniquely determined by $\psi$ because $N_{2}$ is dense in $N_{3}$.)

Proof. As $\Gamma[+](\alpha)$ is a compact subsemiring in which $\alpha$ is a multiplicative unit (Lemma 2), it is a consequence of [6], Theorem 7 that $K[+](\alpha)$ is totally disconnected, and hence 0-dimensional (Theorem 3.5 of [3]). Because $K[+](\alpha)$ is a monothetic additive group with generator $\alpha^{\prime}=\alpha+e$, where $e$ is the unit of $K[+](\alpha)$ ([5], Theorem 3.1.2 or [2], Theorems 3.2 and 3.4), it follows from Theorem 25.16 of [3] that $(K[+](\alpha),+)$ is topologically isomorphic with a cartesian product $\chi_{\rho \in P} A_{\rho}$ where for each $\rho$ in $P, A_{\rho}$ is either the trivial group with one member or the group $Z\left(\rho^{r}\right)$ of residues modulo $\rho^{r_{\rho}}$ for some integer $r_{\rho} \geqq 1$ or the group $\Delta_{\rho}$. (If $K[+](\alpha)$ is finite then it is cyclic and the result follows from Theorem 17, Chapter III of [9].) In what follows we shall assume that $K[+](\alpha)$ is identical with $\mathrm{X}_{\rho \in P} A_{\rho}$. For each $\rho \in P$ we introduce a multiplication $\circ$ on $A_{\rho}$ as the natural ring multiplication and denote by $\mathbf{l}_{\rho}$ its multiplicative unit. We give $\mathrm{X}_{\rho \in P} A_{\rho}$ the coordinate-wise multiplication, for which the element $\beta$, whose coordinate in $A_{\rho}$ is $1_{\rho}$ for all $\rho$, is a multiplicative unit. It is clear that for each $\rho \in P$ there is a continuous additive homomorphism $\phi_{\rho}^{\prime}$ from $\Delta_{\rho}$ onto $A_{\rho}$ with $\phi_{\rho}^{\prime}\left(1_{\rho}\right)=\mathbf{1}_{\rho}$. (If $A_{\rho}$ is isomorphic with $Z\left(\rho^{r_{\rho}}\right)$, put

$$
\phi_{\rho}^{\prime}(x)=x_{0}+x_{1} \rho+\cdots+x_{r_{\rho}-1} \rho^{r_{\rho}-1}
$$


for each $x=\left(x_{0}, x_{1}, x_{2}, \cdots\right)$ in $\Delta_{\rho}$.) If for each $x$ in $N_{1}$ we let $x_{\rho}$ be the coordinate of $x$ in $\Delta_{\rho}$ and we define a function $\phi^{\prime}: N_{1} \rightarrow K[+](\alpha)$ by putting the coordinate of $\phi^{\prime}(x)$ in $A_{\rho}$ equal to $\phi_{\rho}^{\prime}\left(x_{\rho}\right)$ for all $\rho \in P$ and all $x$ in $N_{1}$, then $\phi^{\prime}$ is clearly a continuous additive homomorphism with $\phi^{\prime}(u)=\beta$ and $\phi^{\prime}\left(N_{1}\right)=K[+](\alpha)$. The mapping $f: K[+](\alpha) \rightarrow K[+](\alpha)$ given by $f(x)=\alpha^{\prime} \circ x$ is a continuous additive homomorphism and $f(\beta)=\alpha^{\prime} \circ \beta=\alpha^{\prime}$. Also $f(K[+](\alpha))$ is closed and contains $0[+]\left(\alpha^{\prime}\right)$ and hence its closure $\Gamma[+]\left(\alpha^{\prime}\right)=K[+](\alpha)$; hence $f$ maps $K[+](\alpha)$ onto $K[+](\alpha)$. We now see that the mapping $\phi: N_{1} \rightarrow K[+](\alpha)$ given by $\phi(x)=f\left(\phi^{\prime}(x)\right)$ is a continuous additive homomorphism of $N_{1}$ onto $K[+](\alpha)$ for which

$$
\phi(u)=f\left(\phi^{\prime}(u)\right)=f(\beta)=\alpha^{\prime} .
$$

We extend $\phi$ to become $\Phi: N_{3} \rightarrow \Gamma[+](\alpha)$ by putting $\Phi(n)=n \alpha$ if $n \in N_{2}$. It is not difficult to show that $\Phi$ is a continuous additive homomorphism of $N_{3}$ onto $\Gamma[+](\alpha)$. (However the proof must be split into two cases according as $\Gamma[+](\alpha) \backslash K[+](\alpha)$ is infinite or finite, and use must be made of the characterization of $\Gamma[+](\alpha)$ in either [5], Theorems 3.1.7 and 3.1 .6 respectively or in $[2]$, Theorem 5.6 .)

Finally, we put $\Psi(y, x)=\Phi(y) \cdot x$ (where $\cdot$ is multiplication in $T$ ) for each $y$ in $N_{3}$ and $x$ in $S$. For each $x$ in $S$,

$$
\Psi\left(N_{3} \times\{x\}\right) \subset \Phi\left(N_{3}\right) \cdot x=\Gamma[+](\alpha) \cdot x=\Gamma[+](\alpha x)=\Gamma[+](x) \subset S,
$$

and so $\Psi$ maps $N_{3} \times S$ into $S$. Clearly $\Psi$ is continuous and, for all $n$ in $N_{2}$,

$$
\Psi(n, x)=\Phi(n) \cdot x=n \Phi(1) \cdot x=(n \alpha) \cdot x=n(\alpha \cdot x)=n x=\psi(n, x) .
$$

We now show that (iii) $\Rightarrow$ (i).

Lemma 6. Let $S$ be an additively commutative and additively $\Gamma$-compact semiring for which there is a continous extension' $\Psi: N_{3} \times S \rightarrow S$ of $\psi: N_{2} \times S \rightarrow S$ defined by $\psi(n, x)=n x$. Then $S$ can be embedded in an additively commutative and additively $\Gamma$-compact semiring with multiplicative unit.

Proof. We first adjoin an element $\gamma$ as an isolated point to $S$ so that $\gamma$ is an additive unit and a multiplicative zero for $S^{\prime}=S \cup\{\gamma\}$; then $S^{\prime}$ is an additively $\Gamma$-compact semiring. We also adjoin an element 0 as an isolated point to $N_{3}$ so that 0 is an additive unit and a multiplicative zero for $N_{4}=N_{3} \cup\{0\}$; then $N_{4}$ is a compact semiring. We extend $\Psi$ to $N_{4} \times S^{\prime}$ by putting $\Psi(0, x)=\Psi(y, \gamma)=\gamma$ for all $x$ in $S^{\prime}, y$ in $N_{4}$; then $\Psi$ is continuous on $N_{4} \times S^{\prime}$. Let $T=N_{4} \times S^{\prime}$ and define addition $*$ and multiplication o on $T$ by

$$
\begin{gathered}
\left(y_{1}, x_{1}\right) *\left(y_{2}, x_{2}\right)=\left(y_{1}+y_{2}, x_{1}+x_{2}\right), \\
\left(y_{1}, x_{1}\right) \circ\left(y_{2}, x_{2}\right)=\left(y_{1} y_{2}, x_{1} x_{2}+\Psi\left(y_{1}, x_{2}\right)+\Psi\left(y_{2}, x_{1}\right)\right) .
\end{gathered}
$$


Then clearly $*, \circ$ are continuous and $*$ is associative and commutative. To complete the proof that $T$ is a semiring we first note that, for all $x_{1}$, $x_{2}$ in $S^{\prime}$ and $y_{1}, y_{2}$ in $N_{4}$,

(a) $\left[\Psi\left(y_{1}, x_{1}\right)\right] \cdot x_{2}=\Psi\left(y_{1}, x_{1} x_{2}\right)=x_{1} \cdot\left[\Psi\left(y_{1}, x_{2}\right)\right]$;

(b) $\Psi\left[y_{1}, \Psi\left(y_{2}, x_{1}\right)\right]=\Psi\left(y_{1} y_{2}, x_{1}\right)$;

(c) $\Psi\left(y_{1}, x_{1}+x_{2}\right)=\Psi\left(y_{1}, x_{1}\right)+\Psi\left(y_{1}, x_{2}\right)$;

(d) $\Psi\left(y_{1}+y_{2}, x_{1}\right)=\Psi\left(y_{1}, x_{1}\right)+\Psi\left(y_{2}, x_{1}\right)$.

(These properties are clear for all $x_{1}, x_{2}$ in $S^{\prime}$ and $y_{1}, y_{2}$ in $N_{2} \cup\{0\}$ because $\Psi$ is an extension of $\psi$. The results follow from the continuity of $\Psi$ and the fact that $N_{2}$ is dense in $N_{3}$.) It is a simple matter to use (a)-(d) and the commutativity of + to check the distributive laws and the associativity of 0 .

That $T$ is additively $\Gamma$-compact follows because

$$
\begin{aligned}
\Gamma[*](y, x) & =\{n(y, x) \mid n \geqq 1\}^{-} \\
& =\{(n y, n x) \mid n \geqq 1\}^{-} \subset \Gamma[+](y) \times \Gamma[+](x) .
\end{aligned}
$$

Also, $(1, \gamma)$ is a multiplicative unit for $T$ since

$$
\begin{aligned}
(y, x) \circ(1, \gamma) & =(y \cdot 1, x \gamma+\Psi(y, \gamma)+\Psi(1, x)) \\
& =(y, \gamma+\gamma+x)=(y, x)=(1, \gamma) \circ(y, x) .
\end{aligned}
$$

Finally we note that $\{0\} \times S$ is a subsemiring which is topologically isomorphic with $S$ since

$$
\begin{aligned}
\left(0, x_{1}\right) *\left(0, x_{2}\right) & =\left(0+0, x_{1}+x_{2}\right)=\left(0, x_{1}+x_{2}\right), \\
\left(0, x_{1}\right) \circ\left(0, x_{2}\right) & =\left(0 \cdot 0, x_{1} x_{2}+\Psi\left(0, x_{2}\right)+\Psi\left(0, x_{1}\right)\right) \\
& =\left(0, x_{1} x_{2}+\gamma+\gamma\right)=\left(0, x_{1} x_{2}\right) .
\end{aligned}
$$

When $S$ is compact we have the following result.

THEOREM 3. Let $S$ be a compact additively commutative semiring. Then the following are equivalent.

(i) $S$ can be embedded in a compact additively commutative semiring with multiplicative unit.

(ii) $S$ can be embedded in an additively $\Gamma$-compact semiring with multiplicative left unit.

(iii) There is a continuous extension $\Psi: N_{3} \times S \rightarrow S$ of the mapping $\psi: N_{2} \times S \rightarrow S$ defined by $\psi(n, x)=n x$.

(iv) The mapping $\psi: N_{2} \times S \rightarrow S$ is uniformly continuous.

Proof. As $S$ is compact, the semiring $T$ constructed in Lemma 6 is compact; hence the equivalence of (i), (ii) and (iii). Because $N_{3} \times S$ is a compact Hausdorff space, it can be regarded as a uniform space (see Theorem 
1, page 225 of [1]). Then if $N_{2} \times S$ is given its relative uniformity as a subset of $N_{3} \times S$, the equivalence of (iii) and (iv) follows from Corollary 2 to Theorem 2, page 228 of [1].

Our analysis includes the embedding of rings in rings with unit as a special case.

If $S$ is any topological ring and $M$ is the ring of integers then $S$ can be embedded in the product ring $M \times S$, which is locally compact when $S$ is; the construction is given in [4], page 49, and is similar to that in Theorem 1 .

In an additively $\Gamma$-compact ring, $\Gamma[+](x)$ is a group and so is identical with $K[+](x)$. (This follows immediately from the Corollary to Theorem 1.1.10 of [5]. Alternatively, $\Gamma[+](x)$ contains an additive idempotent (the additive unit of $K[+](x))$; this idempotent must be the additive unit of the ring and so it follows from [8], Theorem 3.2 that $\Gamma[+](x)$ is a group.) In this case we have the following analogue of Lemma 5 .

LeMma 7. Let $S$ be an additively $\Gamma$-compact subring of a topological semiring $T$ with multiplicative left unit $\alpha$ such that $\Gamma[+](\alpha)$ is compact. Then there is a continous extension $\bar{\chi}: N_{1} \times S \rightarrow S$ of the mapping $\chi:\{n u \mid n \geqq 1\} \times S \rightarrow S$ defined by $\chi(n u, x)=n x$.

Proof. Let $\bar{\chi}$ be the restriction to $N_{1} \times S$ of the function $\Psi: N_{3} \times S \rightarrow S$ defined in Lemma 5. Then for each positive integer $n$ and each $x$ in $S$,

But

$$
\bar{\chi}(n u, x)=\Phi(n u) \cdot x=n \Phi(u) \cdot x=\left(n \alpha^{\prime}\right) \cdot x=n\left(\alpha^{\prime} x\right) .
$$

$$
\alpha^{\prime} x=(\alpha+e) x=\alpha x+e x=x+e x,
$$

where $e$ is the additive identity of $K[+](\alpha)$. Now

and

$$
e x+e x=(e+e) x=e x
$$

$$
e x \in K[+](\alpha) \cdot x=K[+](\alpha x)=K[+](x)
$$

so that $e x$ is the additive identity of $K[+](x)$. However $K[+](x)=\Gamma[+](x)$ because $S$ is a ring and so $x+e x=x$. Thus $\bar{\chi}(n u, x)=n x$ which means that $\bar{\chi}$ is an extension of $\chi$.

Conversely, if $S$ is an additively $\Gamma$-compact ring for which $\bar{\chi}$ of Lemma 7 exists, then we can construct an additively $\Gamma$-compact ring on $N_{1} \times S$ (using $\bar{\chi}$ in place of the $\Psi$ of Lemma 6), and $S$ is topologically isomorphic with $\{\varepsilon\} \times S$ where $\varepsilon$ is the additive unit of $N_{1}$. Also, if $\gamma$ is the additive unit of $S,(u, \gamma)$ is a multiplicative unit for the ring $N_{1} \times S$.

Hence we have the following results.

THEOREM 4. Let $S$ be an additively $\Gamma$-compact ring. Then the following are equivalent. 
(i) $S$ can be embedded in an additively $I$-compact ring with unit.

(ii) $S$ can be embedded in an additively $\Gamma$-compact semiring with left unit.

(iii) There is a continuous extension $\bar{\chi}: N_{1} \times S \rightarrow S$ of the mapping $\chi:\{n u \mid n \geqq 1\} \times S \rightarrow S$ defined by $\chi(n u, x)=n x$.

THEOREM 5. Let $S$ be a compact ring. Then the following are equivalent.

(i) $S$ can be embedded in a compact ring with unit.

(ii) $S$ can be embedded in an additively $\Gamma$-compact semiring with left unit.

(iii) There is a continuous extension $\bar{\chi}: N_{1} \times S \rightarrow S$ of the mapping $\chi:\{n u \mid n \geqq 1\} \times S \rightarrow S$ defined by $\chi(n u, x)=n x$.

(iv) The mapping $\chi:\{n u \mid n \geqq 1\} \times S \rightarrow S$ is uniformly continuous.

\section{References}

[1] N. Bourbaki, Topologie générale (Actualités scientifiques et industrielles no. 1142, 1961).

[2] E. Hewitt, 'Compact monothetic semigroups', Duke Math. J. 23 (1956), 447-457.

[3] E. Hewitt and K. A. Ross, Abstract harmonic analysis, Vol. 1 (Springer-Verlag, Berlin, 1963).

[4] A. G. Kurosh, Lectures on general algebra (Chelsea, New York, 1963).

[5] A. B. Paalman-de Miranda, Topological semigroups (Mathematisch Centrum, Amsterdam, 1964).

[6] J. Selden, Theorems on topological semigroups and semirings (Doctoral Dissertation, University of Georgia, 1963).

[7] J. Selden, 'A note on compact semirings' Proc. Amer. Math. Soc. 17 (1966), 882-886.

[8] A. D. Wallace, 'The structure of topological semigroups', Bull. A mer. Math. Soc. 61 (1955), $95-112$.

[9] H. Zassenhaus, The theory of groups (2nd ed., Chelsea, New York, 1958).

\section{University of Adelaide}

South Australia 